\title{
Design thinking aplicado al mejoramiento de las competencias ciudadanas en universitarios: voto popular
}

\section{Design thinking applied to the improvement of citizen competences in university students: popular vote}

\author{
Cristian Hernández-Gil ${ }^{1}$ \\ Jaime Alexis Núñez-López ${ }^{2}$ \\ Recibido: febrero 02 de 2020 \\ Aceptado: junio 02 de 2020
}

\begin{abstract}
Resumen
El objetivo de esta investigación, fue aplicar la técnica de pensamiento de diseño para mejorar las habilidades de pensamiento social dentro de las competencias ciudadanas, de los estudiantes de un programa de Administración de Empresas colombiano. Se realizó un estudio de corte cualitativo, de tipo descriptivo, con 48 estudiantes de últimos semestres, empleando la metodología del design thinking en actividades dentro del aula de clase. En este caso, se trabajó con dos objetos: un dado y un teléfono, y una iniciativa: sensibilización hacia el ejercicio consciente del voto. Los artefactos creados, en total doce, fueron sometidos a criterios de evaluación, especialmente, la capacidad del mismo para que a través de su funcionamiento se le diera respuesta a la pregunta problema. Los diferentes artefactos, cuya función principal es la sensibilización del ejercicio al voto popular, se diseñaron a partir de los principios de: solidaridad, iniciativa e indagación. Se concluye que la aplicación de esta metodología promueve el desarrollo creativo de los estudiantes, en la participación de los mecanismos para ejercer control ciudadano.
\end{abstract}

Palabras clave: pensamiento de diseño, competencias ciudadanas, aprendizaje, innovación.

\begin{abstract}
The objective of this research was to apply the design thinking technique to improve social thinking skills inside the citizen competences of the students of a Colombian Business Administration program. A qualitative and descriptive study with 48 students of last semesters was carried out, implementing the design thinking methodology in activities inside the classroom with them, In. this case, we worked with two objects: a die and a telephone, and an initiative: awareness-raising towards the conscious exercise of voting. The twelve artifacts created, were subjected to evaluation criteria, especially their capacity so that the problem question could be answered through their operation. The different artefacts built, whose main function is to raise awareness of the exercise of popular vote, were designed based on the principles of solidarity, initiative and inquiry. It was concluded that the application of this methodology promotes the creative development of the students, applied to the participation of the mechanisms to exercise citizen control.
\end{abstract}

Keywords: design thinking, citizen competitions, learning, innovation.

1 Administrador de Empresas, Magíster en Ciencias de la Educación, Universidad de la Amazonia, Florencia, Colombia. E-mail: cris. hernandez@udla.edu.co. ORCID: 0000-0001-6512-2453

2 Estudiante de Licenciatura en Ciencias Sociales, Universidad de la Amazonia, Florencia, Colombia. E-mail: jai.nunez@udla.edu.co. ORCID: 0000-0002-1896-2828 


\section{Introducción}

Las competencias ciudadanas se enmarcan dentro de las habilidades que debe adquirir todo individuo para el ejercicio de su profesión. Tanto administradores, como abogados, entre otros profesionales, reciben formación universitaria, con el fin de entender y apropiarse de su papel como ciudadanos, participando activamente de las relaciones que se ejercen dentro del ente territorial donde reside cada uno.

Las competencias ciudadanas, se definen como: los conocimientos y habilidades que posibilitan la construcción de marcos de comprensión del entorno, los cuales promueven el ejercicio de la ciudadanía y la coexistencia inclusiva dentro del marco que propone la Constitución Política de Colombia. El ejercicio de la ciudadanía va mucho más allá del entendimiento y aplicación de los derechos y deberes. Un ciudadano competente es aquel que, además de poseer un conocimiento de su entorno social y político, tiene la capacidad de reflexionar sobre los ejes problemáticos en los que se enmarca la ciudadanía y su colectivo, y frente a ello postula alternativas que minimizan los impactos negativos frente al bienestar comunitario.

Según el Ministerio de Educación Nacional, MEN (2012), dentro de sus lineamientos para la formación de competencias ciudadanas, se reconoce que estas se desarrollan en todo el ciclo educativo de cualquier persona. La idea fundamental es que cuando cada colombiano inicie su vinculación al mundo laboral, sea capaz de entender el contexto, valorar la diversidad cultural, así como reconocer sus derechos individuales y colectivos, para actuar de manera constructiva y propositiva frente al respeto por las libertades de los demás.

Según el Instituto Colombiano para la Evaluación de la Educación, ICFES (2018), las competencias ciudadanas se enmarcan dentro de la evaluación de competencias genéricas en el examen Saber Pro. Siguiendo este parámetro, es importante recalcar que la formación en este tipo de competencias debe desarrollarse en todas las áreas del conocimiento, debido a su carácter transversal. Así se espera que las competencias en mención, articuladas a los estándares aplicados a la educación básica y media, continúen formándose en la educación superior en cada pregrado, en un nivel más complejo. Así lo recalca Acosta-Varela (2016), indicando que la democracia debe ser impulsada con la ciudadanía en todas las instituciones, desde el conglomerado familiar, la escuela, hasta los entes gubernamentales, sin descuidar el valor de lo público. Este último es el único espacio donde los sujetos sociales se pueden integrar para restituir las diferencias, injusticias y conflictos enmarcados en el quehacer ciudadano (Redon, 2016).

Según el ICFES (2018), para ser un ciudadano competente, el individuo debe desarrollar habilidades tanto cognitivas como emocionales y comunicativas. Las competencias cognitivas buscan indagar en el estudiante su nivel de conocimiento sobre los fundamentos para: convivir dentro de la ciudadanía, ser un participante democrático y valorar el pluralismo. Además, se evalúa su razonamiento y análisis frente a situaciones de convivencia social. En últimas, se busca que el estudiante reconozca la Constitución Nacional, sus alcances y limitaciones para luego utilizarla en los contextos donde el asume su papel de ciudadano. En el módulo de competencias ciudadanas, se evalúan cuatro habilidades cognitivas: pensamiento social, argumentación, multiperspectivismo y pensamiento sistémico.

El Programa de Administración de Empresas, de la Universidad de la Amazonia, ha obtenido un nivel de desempeño relativamente bajo, en el módulo de competencias ciudadanas del examen Saber Pro, durante los tres últimos años, alcanzando promedios por debajo de la media nacional e institucional. Además, los promedios individuales del programa han tenido un nivel de dispersión que sobrepasa la media nacional de 30 . En este sentido, el problema de investigación radica en el 
bajo desempeño de los estudiantes, en el módulo de competencias ciudadanas.

Las causas de este desempeño aún no se han podido establecer de manera formal. Los estudiantes, actualmente reciben formación relacionada con competencias ciudadanas, a través de tres asignaturas. En primer semestre, está la asignatura Sociedades, donde aprenden lo relacionado con los fundamentos del código de comercio. En séptimo semestre, existe una asignatura denominada Constitución y Democracia, y en noveno semestre, se finaliza la formación en ciudadanía a través de la asignatura Ética Empresarial.

En un estudio reciente sobre el análisis del contexto para la formación en administración de empresas, se comprobó que para el componente de formación integral que incluye las asignaturas mencionadas: el 50,92\% de los estudiantes, refiere que la metodología más utilizada por los docentes son los talleres individuales y grupales, seguida de los seminarios y la clase magistral. La percepción de satisfacción de los estudiantes frente al componente sociohumanístico, se encuentra distribuida de la siguiente manera: el $42.22 \%$ de la población indica una percepción buena frente a la formación, el 32.19\% indica una percepción aceptable, mientras que el $5.8 \%$ y $2.9 \%$ manifiestan una percepción insuficiente y deficiente, respectivamente (Andrade-Adaime et al., 2019).

La investigación planteó como objetivo principal, aplicar la técnica de pensamiento de diseño o design thinking, para mejorar las habilidades de pensamiento social dentro de las competencias ciudadanas, de los estudiantes del Programa de Administración de Empresas, de la Universidad de la Amazonia. Teniendo en cuenta el desarrollo de las elecciones territoriales al momento del trabajo de campo, se trabajó el ejercicio al voto, como uno de los fundamentos del pensamiento social para lograr la competencia en ciudadanía. Lo anterior lleva a plantear dos perspectivas: la primera relacionada con la apropiación del conocimiento del individuo sobre los fundamentos que rigen los derechos y deberes constitucionales; y la segunda relacionada con la reconstrucción de la realidad social y el análisis de sus elementos en una situación dada, es decir, las formas en las que los estudiantes construyen soluciones a partir de las problemáticas de su entorno inmediato.

\section{Marco teórico y metodología}

2.1 Formación en competencias ciudadanas en pregrado

Para Benedicto y Morán (2002), el concepto de ciudadanía tiene una perspectiva compleja y multidimensional, que abarca tres dimensiones: los principios legales y constitucionales que vinculan al sujeto con la ciudadanía; la reconstrucción cultural que todos los días se desarrolla a través de las prácticas de ciudadanía dentro de una comunidad, es decir, la praxis de la costumbre social; y el carácter del ejercicio colectivo que agrupa las relaciones recíprocas entre el gobierno y sus ciudadanos. Estas consideraciones llevan a definir que la formación en ciudadanía, se hace desde el ejercicio intencional de la historia de la vida pública y privada de: las identidades, símbolos, manifestaciones y creencias que conforman las expresiones de los miembros de la ciudadanía (Henao-Escovar et al., 2007).

En el caso colombiano, la formación ciudadana en los acuerdos constitucionales del año 1991, se entendió como una preocupación nacional evidenciada en la necesidad de edificar una democracia participativa en la sociedad de ese entonces. En términos históricos, la sociedad y el Estado venían presentando un deterioro en sus relaciones, gracias a: la prolongación del conflicto interno, la falta de legitimidad de las instituciones, y la creciente indiferencia de los ciudadanos hacia el ejercicio político y la vida pública (Pinilla-Díaz \& Torres-Azocar, 2006).

Por su parte, De Sousa-Santos (2005), atribuyéndole el compromiso a las universidades sobre la formación en ciudadanía, recalca que los claustros 
de educación superior deben, a través de los procesos académicos, recuperar el sentido político de su estructura social, y así reconocer la pluralidad de grupos sociales que allí se establecen, para contribuir a consolidar las instancias democráticas de la sociedad. Es meritorio reconocer que la mayoría de los estudiantes, no solo llegan a las universidades a adquirir competencias disciplinares en su área del saber o profesional, además llegan con la intención de formarse como ciudadanos competentes que promuevan el ejercicio de la democracia y el desarrollo de las sociedades.

Para que este escenario se dé, es importante entender las formas en las que se da el ejercicio ciudadano por parte de este público universitario. Sus principales expresiones tienden a ser más sociales y culturales, las cuales son utilizadas para transmitir sus preocupaciones y experiencias individuales, en espacios públicos que enriquecen la vida académica. Para Henao et al. (2007), la formación ciudadana de los estudiantes en las universidades puede desarrollarse a través de mecanismos que los hagan más visibles, como el uso de medios de comunicación. Así mismo, desde la investigación, en los diferentes semilleros universitarios, se puede orientar a los futuros profesionales hacia la producción de conocimientos sobre las problemáticas nacionales. De esta manera, se pueden constituir en el germen de procesos organizativos que generen prácticas ciudadanas para fortalecer los vínculos entre la universidad y la sociedad.

Zambrano (2018), indagó sobre la incidencia de las prácticas pedagógicas constructivistas, como los esquemas lúdicos en el aula de clase, sobre el desarrollo de las competencias ciudadanas en estudiantes de la Universidad del Magdalena. El autor infiere que la aplicación de estas prácticas, logra mejores resultados de aprendizaje que las prácticas expositivas relacionadas con clases magistrales. Lo anterior fue explicado por tres razones: el sentido constructivista de la práctica que lleva al estudiante a ser protagonista de su propia formación, la etapa formativa de los estudiantes que ya habían superado más de la mitad de las asignaturas del plan de estudios, y la relación de la competencia ciudadana con la habilidad cognitiva. Es decir, la evaluación de la adquisición de las competencias ciudadanas está dada por la integración de elementos que ponen a prueba la práctica del sujeto en un entorno: se forma mejor desde la actuación (Calderíus-Fernández \& Martínez-Sánchez, 2013). La vivencia lleva al sujeto, como ciudadano, a tener un pensamiento social, sistémico, argumentado, y con múltiples perspectivas, siendo más empático.

\subsection{Pensamiento de diseño como instrumento para formar}

El pensamiento de diseño o design thinking, es una metodología creada a comienzos de siglo XXI en la Universidad de Stanford, cuyo principal fundamento es entender al individuo, sus interacciones respecto al entorno y sus necesidades, y sobre ello plantear soluciones que lo satisfagan (Frías, 2019). Su efectividad radica en la posibilidad de crear valor durante el proceso y en sus resultados (Kimbell, 2011). Esto supone la oportunidad para mejorar las condiciones de vida de la sociedad, teniendo presente: sus deseos, demandas y anhelos, adicional al establecimiento de problemáticas como factor negativo. El pensamiento de diseño tiene cinco fases, distribuidas a través de un eje secuencial que permite identificar un problema y darle solución, a través de: la inventiva, la creatividad y la innovación.

Para emplear esta metodología, Dorst (2011), argumenta que existen dos maneras: 1. Desde la deducción como el proceso de pasar de saber "qué" y "cómo" al "resultado", y 2. Desde la inducción como el proceso de pasar de saber "qué" y "resultado" a posibles opciones de "cómo". Esta metodología es generalmente usada en escuelas de negocios, para la creación de nuevos productos.

En algunos claustros universitarios, existen espacios académicos que brindan a los estudiantes la oportunidad de desarrollar su creatividad, empleando diversas estrategias de enseñanza 
que empoderan estas capacidades de innovación en los individuos. La Universidad Tecnológica del Perú, utiliza modelos de creación integrados en su programa de MBA, a través de la metodología Taller Lego Serius Play para potenciar su experiencia en el proceso de formación y transformación estratégica, generando un proceso vivencial que logra el desarrollo de competencias de liderazgo, pensamiento, comunicación y resolución de problemas en organizaciones y equipos. En España, la Escuela de Organización Industrial en sus programas de MBA, tiene un módulo denominado Innovación Aplicada, en donde el estudiante entrena su creatividad con técnicas de design thinking, con el fin de aportar nuevas soluciones a los problemas actuales de su entorno económico. En Dinamarca, la escuela de negocios Kaos Pilot, es un referente en innovación social, que promueve la integración de: la experimentación, la exploración, y la experiencia.

En Estados Unidos, el Instituto Tecnológico de Massachusetts, a través de su Media Lab, los estudiantes desarrollan proyectos que integran: la tecnología, el mundo multimedia y el diseño, a fin de estimular la innovación, enseñando a inventar y reinventar herramientas usadas en la gestión de los negocios. En Canadá, la Universidad de Toronto aplica las metodologías Integrative Thinking y Business Design, como instrumentos para crear soluciones creativas.

El design thinking, se puede proponer como: una forma cognitiva resultante del ejercicio prospectivo de alguna disciplina relacionada con el diseño, o como una herramienta utilizada por la gestión organizacional centrada en las personas. En la primera se establecen prototipos, y para la segunda se definen artefactos: es decir, los resultados de una acción deliberada que produce valor agregado a una solución específica (Córdoba-Cely et al., 2016). Los autores reconocen que este resultado se puede considerar práctico, si su función es crear valor (efectividad), o teórico si su finalidad es encontrar la verdad (adaptación al contexto). En la presente investigación se implementó el segundo tipo, por las condiciones cognitivas de los estudiantes y por el interés científico de los investigadores. Los estudiantes aprenden de manera más eficaz cuando asumen retos contextualizados, originados en su propia realidad. En este sentido, existe una relación proporcional directa entre la formación de ciudadanía en las universidades y el uso del pensamiento de diseño. En ambos casos, se hace necesario un aprendizaje basado en escenarios, donde el estudiante hace una inmersión a la realidad social y busca mejorarla, desde: la costumbre, sus principios, sus ideales y lo establecido en los esquemas constitucionales.

\subsection{El examen Saber Pro en competencias ciudadanas}

Según Ascolfa (2018), los resultados en el módulo de competencias ciudadanas del año 2017, en todos los grupos de referencia, se caracterizaron por una disminución con respecto al año anterior, de 8,5 puntos, aproximadamente: el puntaje promedio a nivel nacional, fue de 142 de 300 puntos posibles. Esta tendencia es marcada en todos los grupos de referencia, donde las mayores variaciones en el puntaje, se encontraron en: Psicología $(-9,8)$, Recreación y deporte $(-9,8)$, Enfermería $(-10,4)$ y Ciencias militares $(-10,4)$. El grupo de referencia Administración y afines, en los resultados de este módulo, tuvo una disminución en su puntaje promedio de 9,3 puntos por debajo del puntaje del año 2016, llegando a 137 puntos, indicador inferior a la media nacional dado por el ICFES (150), y al puntaje promedio obtenido por todos los estudiantes del país (142), en el año 2017.

Además, según los niveles de desempeño en el módulo de competencias ciudadanas, más del $60 \%$ de los estudiantes del país están ubicados en los niveles básicos: 1 y 2 , donde se encuentra todos los estudiantes del grupo de referencia de Administración y afines. Los mejores desempeños, se presentan en: Humanidades, Economía y Medicina, quienes se ubican en los niveles de desempeño 3 y 4 (Ascolfa, 2018). 
La tendencia en el desempeño de los estudiantes a nivel nacional y los del programa de Administración de Empresas de la Universidad de la Amazonia, es similar. Esto justifica el desarrollo de la presente investigación, con miras a fortalecer estos desempeños, para mejorar los indicadores de calidad del programa. Lo anterior permitirá formar no solo buenos administradores, sino también ciudadanos idóneos, que participen activamente en las decisiones sociales y políticas de su contexto.

\subsection{Diseño metodológico}

Se adelantó un estudio de enfoque cualitativo, cuya principal característica fue entender a través de la aplicación de una técnica de innovación, la apropiación de las competencias ciudadanas que adquieren los estudiantes; es decir, el nivel de la habilidad de pensamiento social, específicamente, su conocimiento frente al ejercicio del voto. No se trata de medir ni de cuantificar una variable. En este caso, se busca caracterizar: los fenómenos, cambios o disposiciones nuevas que se generan cuando se implementa una estrategia pedagógica en la formación de administradores de empresas.

La tipología investigativa seleccionada fue la descripción, siendo parte fundamental de este proceso. El método de estudio, fue el pensamiento de diseño o design thinking. El proceso investigativo permitió detallar qué sucede con los estudiantes, cuando se enfrentan a diseñar soluciones para mejorar sus procesos de aprendizaje. A continuación, se describen las fases del método y su implementación:

Fase I o Entendimiento del entorno. Está conformada por dos actividades: empatizar con el sujeto y definir el problema. Inicialmente el método conduce a los estudiantes a entender el eje problemático a través de un reto. Este consistía en hacer reflexionar al grupo sobre cómo sensibilizar a los ciudadanos sobre la importancia de ejercer el voto a conciencia. Para Carvajal (2016a), citando al MEN (2014), explorar el medio implica aprender a través de la vida y todo lo que en ella se establece; es decir, los objetos físicos naturales y los construidos por el hombre, las personas, las relaciones entre unos y otras. En este sentido, se definieron doce (12) equipos de trabajo, de cuatro (4) estudiantes cada uno. Primero se analizó el perfil del sujeto al cual debían sensibilizar, para luego a través de una pregunta, identificar el problema que debían solucionar. En esta fase, se utilizó el mapa de empatía como herramienta para categorizar el tipo de público dentro de la ciudadanía, a los cuales debía dirigirse la solución. Adicionalmente, se realizó una revisión documental para analizar tendencias sobre prácticas de generación de conciencia frente al voto en diversos contextos, y se aplicó la técnica de grupos focales para determinar los públicos a trabajar.

Fase II o Exploración de las alternativas. Está compuesta por dos actividades: producir ideas y prototipar soluciones. Luego de identificar el problema a superar, los estudiantes, utilizando herramientas de innovación, como: la hibridación y el pensamiento lateral, empezaron un proceso de ideación de la solución a través de diferentes artefactos. En esta fase creativa, cada uno propuso alternativas que conectaban con: el conocimiento constitucional sobre el ejercicio al voto en Colombia, los saberes empíricos relacionados con ingeniería para la construcción del artefacto y la adecuación de la solución, teniendo en cuenta los elementos demográficos y sicográficos del público objetivo. El producto final de esta fase, fue la construcción del modelo de representación.

Fase III o Materialización de la solución. En este proceso, los estudiantes se enfrentaron a la realidad de sus públicos objetivos, presentándoles el artefacto. Los grupos de trabajo anotaron las observaciones dadas por los sujetos, que manipularon el producto resultado de la fase anterior, y establecieron sus propias conclusiones y recomendaciones. La elección de estos sujetos, se hizo a través de un muestreo no probabilístico usando algunos criterios de selección a juicio de los investigadores. La herramienta de recolección 
de datos en esta fase, fueron los grupos focales con los públicos objetivos, quienes validaron los artefactos.

Los resultados que se presentan en la siguiente sección, parten del principio de la flexibilidad. Podrán variar de acuerdo a las condiciones de cada contexto donde se replique. En ellos no se encontrará: la aplicación sistémica de un formato o cuestionario, el uso de unas actividades mecánicas y la medición de la adquisición de un nuevo conocimiento. Sin embargo, se presentan ideas en dos frentes: i) la importancia de un método de creatividad y aplicación de la innovación, enfocado en un tema específico, puede hacer que el estudiante aprenda y a la vez enseñe; ii) la generación de retos y problemáticas logran llevar al estudiante a integrar sus conocimientos, para que, en este caso, además de aprender a votar a conciencia, perfeccione su proyecto de vida con la generación de artefactos como emprendimientos.

\section{Resultados y discusión}

En la fase I, los equipos de trabajo exploraron los elementos constitucionales relacionados con el ejercicio al voto, según los artículos: 100, 103 al 112, y 258 al 266, de la Constitución Política de Colombia (1991). Esta información se contrastó con el aprendizaje significativo que han desarrollado, a través de su vida: social, académica, familiar y personal, frente al tema. El resultado de este proceso fue un documento escrito, que se analizó a través del software Atlas.ti, donde se encontraron diferentes esquemas semánticos, como se ilustra en la Figura 1.

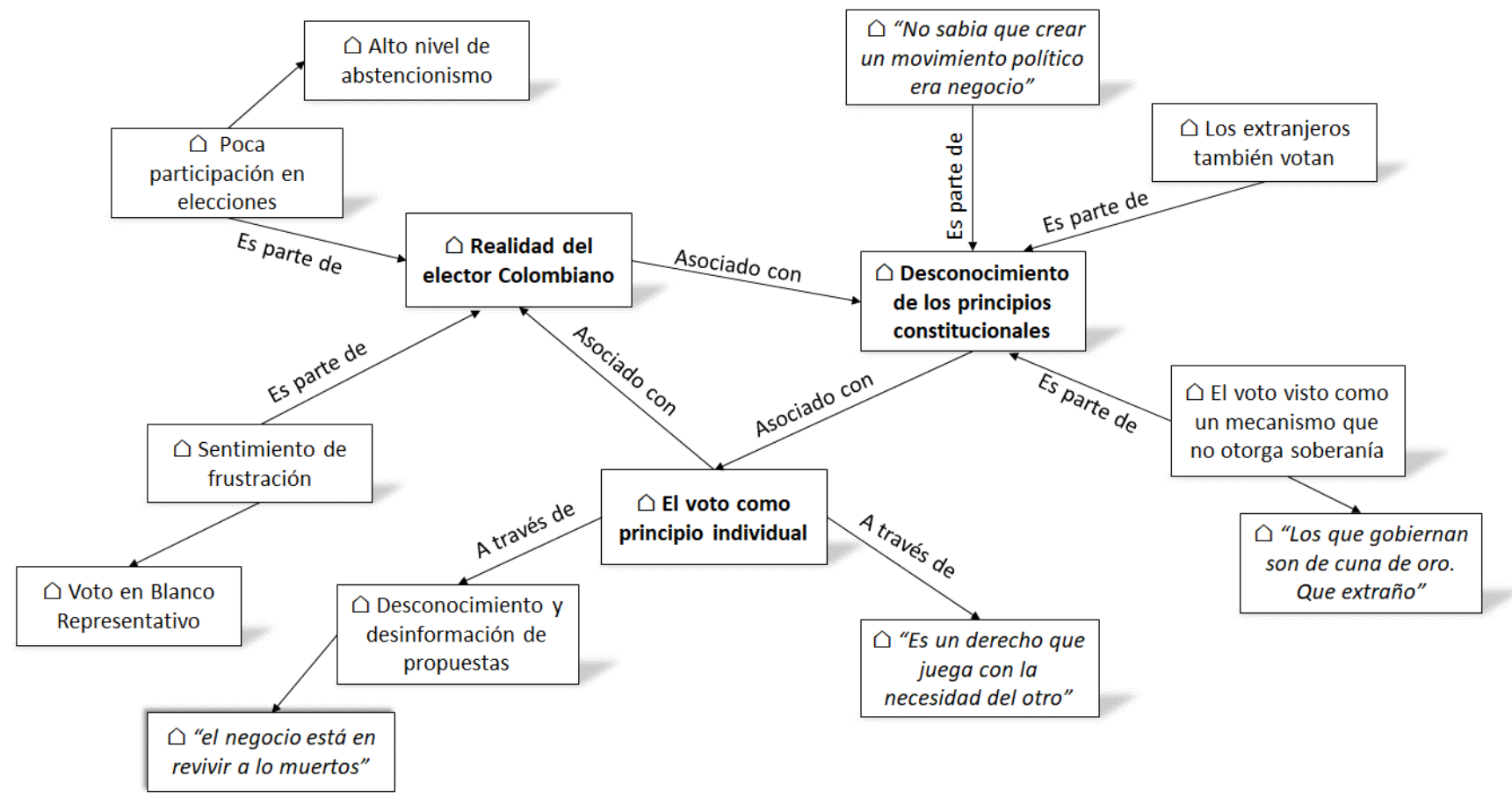

Figura 1. Red semántica del análisis del ejercicio al voto en Colombia.

En el análisis, se constató que los estudiantes explican la existencia de una brecha amplia entre lo expuesto en la constitución y lo evidenciado en la vida política y social de los ciudadanos, durante los procesos electorales de los últimos veinte años. Para ellos, el ejercicio de elegir a sus mandatarios 
no es visto como un derecho en donde predomine la democracia, sino más bien como la oportunidad de mejorar sus condiciones de vida, a través del lucro particular. Se denota un desconocimiento de todas las disposiciones frente al ejercicio al voto, justificado en gran parte por el desinterés en la participación activa de la política en Colombia. Esto se refuerza por la desmotivación que se genera al reconocer que las personas que ganan las elecciones no siempre son las adecuadas, aludiendo a expresiones como: "las elecciones siempre están arregladas", y "quien las gana no es el más capaz, sino el que más tiene para pagar".

Luego se realizaron una serie de grupos focales con los equipos de trabajo, donde se debatió el perfil de las poblaciones que tienen más probabilidad de ser manipuladas, al momento de votar. Antes de participar en los debates, cada equipo elaboró un mapa de empatía, con un perfil propuesto según la indagación previa en el entorno donde se desarrolla. Los estudiantes reconocieron tres tipologías, como se muestra en la Tabla 1.

Tabla 1. Tipología de públicos.

\begin{tabular}{|c|c|c|c|}
\hline $\begin{array}{c}\text { Tipología/ } \\
\text { Característica }\end{array}$ & Campesinos Colombianos & Primíparos electorales & Millennials emancipados \\
\hline Justificación & $\begin{array}{l}\text { Son personas residentes en } \\
\text { zonas rurales, que poco creen } \\
\text { en las propuestas ni en los } \\
\text { candidatos. Debido a su lejanía } \\
\text { con los puestos de votación, no } \\
\text { ejercen su derecho, y si lo hacen, } \\
\text { es porque algún político les } \\
\text { concede el transporte o algún } \\
\text { beneficio. }\end{array}$ & $\begin{array}{l}\text { Son estudiantes que } \\
\text { apenas han cumplido } \\
\text { la mayoría de edad y no } \\
\text { han ingresado a la vida } \\
\text { política. Usualmente, } \\
\text { son manipulados por } \\
\text { las convicciones de sus } \\
\text { padres, profesores y/o } \\
\text { acudientes, para ejercer } \\
\text { el voto. }\end{array}$ & $\begin{array}{l}\text { Son profesionales con edades } \\
\text { entre } 26 \text { y } 35 \text { años, que no han } \\
\text { definido una postura política. } \\
\text { Se abstienen de votar por la no } \\
\text { aceptación de los candidatos que, } \\
\text { según ellos tienen las mismas } \\
\text { características: son incapaces, y } \\
\text { esta debilidad la ocultan tras el } \\
\text { tener recursos. Los que votan, lo } \\
\text { hacen por mejorar su situación } \\
\text { laboral únicamente. }\end{array}$ \\
\hline $\begin{array}{l}\text { ¿Qué piensan y } \\
\text { sienten? }\end{array}$ & $\begin{array}{l}\text { Se sienten incapaces de hacer } \\
\text { algo, desde la política o votando, } \\
\text { para mejorar las condiciones de } \\
\text { vida en el campo. }\end{array}$ & $\begin{array}{l}\text { Piensan que deben } \\
\text { participar votando por } \\
\text { el candidato que les da } \\
\text { oportunidades labo- } \\
\text { rales o estudio. }\end{array}$ & $\begin{array}{l}\text { Se sienten frustrados por saber } \\
\text { que los elegidos siempre son los } \\
\text { mismos. }\end{array}$ \\
\hline ¿Qué ven? & $\begin{array}{l}\text { En general, ven como sus tierras } \\
\text { día a día son menos productivas } \\
\text { y no poseen los recursos para } \\
\text { recuperarlas. }\end{array}$ & $\begin{array}{l}\text { En general, ven } \\
\text { programas de TV que } \\
\text { no están relacionados } \\
\text { con espacios políticos } \\
\text { o de interés público. } \\
\text { Se aíslan de la realidad } \\
\text { nacional, a través del } \\
\text { entretenimiento. }\end{array}$ & $\begin{array}{l}\text { En general, ven: noticias, } \\
\text { programas de opinión, y espa- } \\
\text { cios virtuales, donde se critica el } \\
\text { actuar político de muchos legisla- } \\
\text { dores y/o ejecutivos. }\end{array}$ \\
\hline ¿Qué escuchan? & $\begin{array}{l}\text { En general, a través de la radio, } \\
\text { escuchan noticias de tipo econó- } \\
\text { mico, político y regional. }\end{array}$ & $\begin{array}{l}\text { En general, escu- } \\
\text { chan estaciones de } \\
\text { radio de música y } \\
\text { entretenimiento. }\end{array}$ & $\begin{array}{l}\text { En general, a través de la radio, } \\
\text { escuchan: noticias de tipo econó- } \\
\text { mico, político y regional, aunque } \\
\text { en menor proporción que los } \\
\text { campesinos. }\end{array}$ \\
\hline
\end{tabular}




\begin{tabular}{|c|c|c|c|}
\hline $\begin{array}{c}\text { Tipología/ } \\
\text { Característica }\end{array}$ & Campesinos Colombianos & Primíparos electorales & Millennials emancipados \\
\hline $\begin{array}{l}\text { ¿Qué dicen o } \\
\text { hacen? }\end{array}$ & $\begin{array}{l}\text { Expresiones como: "Todos } \\
\text { son iguales, por eso es que } \\
\text { estamos como estamos"; "Yo } \\
\text { voto por quién me le ayude a } \\
\text { mi hijo a estudiar o me apoye } \\
\text { económicamente". }\end{array}$ & $\begin{array}{l}\text { Expresiones como: "No } \\
\text { tengo ni idea por quién } \\
\text { votar, aún así lo haré así } \\
\text { sea en blanco". } \\
\text { "Mi papá me dijo que } \\
\text { votará por x candidato } \\
\text { pues él me puede } \\
\text { ayudar a conseguir } \\
\text { trabajo". }\end{array}$ & $\begin{array}{l}\text { Expresiones como: "Para que } \\
\text { votamos, de todos modos eso ya } \\
\text { está arreglado". } \\
\text { "Necesito razones para votar } \\
\text { por un verdadero líder social y } \\
\text { ambiental". } \\
\text { "Necesito votar por el candidato } \\
\text { x, ya que si gane tendré contrato } \\
\text { por lo menos por cuatro años". }\end{array}$ \\
\hline $\begin{array}{l}\text { Miedos } \\
\text { principales }\end{array}$ & $\begin{array}{l}\text { Que el gobierno o terceros } \\
\text { expropien sus tierras, que les } \\
\text { toque migrar a la ciudad por } \\
\text { situaciones de pobreza o falta de } \\
\text { oportunidades de trabajo en el } \\
\text { campo. }\end{array}$ & $\begin{array}{l}\text { No tener recursos para } \\
\text { apoyar la economía } \\
\text { familiar. }\end{array}$ & $\begin{array}{l}\text { No tener un empleo estable, y } \\
\text { ser protagonista de impactos } \\
\text { ambientales negativos. }\end{array}$ \\
\hline Aspiraciones & $\begin{array}{l}\text { Tener la capacidad de sacar a su } \\
\text { familia a adelante. }\end{array}$ & $\begin{array}{l}\text { Tener la oportunidad de } \\
\text { emplearse o estudiar. }\end{array}$ & $\begin{array}{l}\text { Tener la capacidad y oportunidad } \\
\text { de desarrollar un empleo por } \\
\text { mérito propio. }\end{array}$ \\
\hline
\end{tabular}

Al final de esta etapa, los equipos, a través de una pregunta reto, definieron el problema que debían afrontar para el desarrollo de la segunda fase. En la Tabla 2, donde E significa equipo, se observan estas problemáticas.

Tabla 2. Problemas reto identificados.

\begin{tabular}{cl}
\hline \multicolumn{1}{c}{ Tipología } & Problemas \\
\hline & $\begin{array}{l}\text { E1: ¿Cómo podemos ayudar a los campesinos a que generen un interés por ejercer el derecho } \\
\text { al voto, sin esperar un beneficio personal? }\end{array}$ \\
& $\begin{array}{l}\text { E4: ¿Cómo podemos ayudar a los campesinos a que tengan una educación y conciencia } \\
\text { ciudadana, a la hora de ejercer el voto? }\end{array}$ \\
$\begin{array}{l}\text { Campesinos } \\
\text { Colombianos }\end{array}$ & $\begin{array}{l}\text { E5: ¿Cómo podemos ayudar a los campesinos a que ejerzan su derecho al voto, teniendo en } \\
\text { Cuenta tienen o no transporte? }\end{array}$
\end{tabular}

E6: ¿Cómo podemos ayudar a los campesinos a que ejerzan de manera correcta el derecho al voto?

E10: ¿Cómo podemos ayudar a los campesinos a que participen activamente en los procesos electorales en el departamento? 


\begin{tabular}{cl}
\hline \multicolumn{1}{c}{ Tipología } & Problemas \\
\hline & $\begin{array}{l}\text { E3: ¿Cómo podemos ayudar a los primíparos electorales a que conozcan sus derechos y } \\
\text { deberes como votantes? }\end{array}$ \\
& $\begin{array}{l}\text { E7: ¿Cómo podemos ayudar a los primíparos electorales a que reconozcan la importancia de } \\
\text { ejercer el voto de manera activa y con conciencia? }\end{array}$ \\
Primíparos & $\begin{array}{l}\text { E8: ¿Cómo podemos ayudar a los primíparos electorales a que tomen el hábito de participar } \\
\text { electorales }\end{array}$ \\
& $\begin{array}{l}\text { E11: ¿Conscientemente de los procesos electorales? } \\
\text { responsable frente a las elecciones? }\end{array}$ \\
& $\begin{array}{l}\text { E12: ¿Cómo podemos ayudar a los primíparos electorales a que conozcan la importancia de } \\
\text { ejercer el voto? }\end{array}$ \\
& $\begin{array}{l}\text { E2: ¿Cómo podemos ayudar a los Millenials emancipados a que busquen y desarrollen motivos } \\
\text { para tener una inclinación política definida, de acuerdo a sus intereses sociales? }\end{array}$ \\
Millennials & \\
emancipados & E9: ¿Cómo podemos ayudar a los Millenials emancipados a que tomen la decisión de sufragar? \\
\hline
\end{tabular}

En la fase dos, los equipos iniciaron un proceso de ideación de las posibles alternativas, que por medio de un artefacto, lograran dar respuesta a la situación problema. Para ello utilizaron la técnica de la hibridación, que consiste en diseñar un elemento o producto nuevo a través de la relación de las características de dos o más ya existentes en la realidad. En este caso, se trabajó con dos objetos: un dado y un teléfono, y una iniciativa: sensibilización hacia el ejercicio consciente del voto. De esta forma afloró la creatividad de cada equipo, quienes utilizaron el tipo de hibridación por agregación. Los artefactos creados, en total doce, fueron sometidos a unos criterios de evaluación, especialmente, la capacidad del mismo, para que con su funcionamiento, se le diera respuesta a la pregunta problema.

Para el caso de los campesinos, se construyeron cinco artefactos, orientados a sistemas de recordación y fuentes de información. Se destacan dos de ellos: un juego que muestra al campesino los dos escenarios que se presentan cuando se vota a conciencia y cuando no se vota en las mismas condiciones; el otro consiste en una máquina que informa de manera visual y auditiva, el perfil y las propuestas de los candidatos. Dentro de la simbología utilizada, se observa que los estudiantes promueven mejorar las competencias ciudadanas en los campesinos, a través de la visualización de imágenes que ellos reconocen de su entorno, así como su interacción con lúdicas que permiten imaginar lo que pasa en diferentes escenarios. Los campesinos, a través de los artefactos, aprenden mejor cuando desarrollan estilos de tipo visual y auditivo, donde no se requieren modelos de representación de alta complejidad tecnológica, sino más bien, de esquemas interactivos con funciones mecánicas o manuales y que se asemejan a elementos que ellos utilizan a diario.

En cuanto a los artefactos para los "primíparos electorales", cinco en total, todos se enfocaron en mostrar la información a través de juegos. La intención era que el elector inicial, tuviera la oportunidad de aprender sus derechos y deberes como ciudadano a través del aprendizaje kinestésico.

Los artefactos para los Millennials, en total dos, tenían como intención reforzar su conocimiento sobre las propuestas de los candidatos. Para los estudiantes, este tipo de público puede alcanzar 
la competencia ciudadana del ejercicio al voto, cuando tienen mayor información sobre las opciones políticas, y cuando esta se presenta de forma organizada y por temas de interés. Por ejemplo, uno de los artefactos, denominado The Box, tiene en cada uno de sus lados los aspectos más importantes para el desarrollo territorial, tales como: salud, educación e infraestructura, entre otros. Al elegir cualquiera de estos lados, a través de un cilindro giratorio, iba desenrollándose un papel con información sobre las propuestas en dichas áreas.

En la fase de validación, los artefactos fueron presentados a un grupo de personas pertenecientes a los públicos segmentados al principio. Haciendo uso de grupos focales, se comprobó una relación de reciprocidad entre las funciones de los artefactos y las necesidades de conocimiento, frente al tema del derecho al voto.

Los campesinos expresaron su preocupación por el campo colombiano, por la falta de apoyo de parte del gobierno y su incapacidad para salir adelante y generar desarrollo rural. La concepción paternalista que se evidencia, sumada al desconocimiento de los principios constitucionales sobre el derecho al voto como mecanismo de participación, permite identificar el principio de la indagación. Ellos aseguran que la ciudadanía se adquiere más allá de conocer propuestas y perfiles de los candidatos, prefiriendo que los artefactos resuelvan parte de sus necesidades de información y de motivación hacia la acción de votar. Esto con el fin de generar una sinergia que promueva el trabajo de políticos y la experiencia del individuo rural, para un verdadero cambio en las relaciones entre: el hombre, la naturaleza y los medios de producción. Lo anterior se evidencia en lo expresado por un campesino: "hasta que no haya un trabajo en equipo, no de unos meses sino de años, hasta el infinito, seguirá existiendo eso que ustedes llaman calentamiento global y deforestación". Es decir, los campesinos adquieren las competencias ciudadanas cuando observan el progreso en la zona rural, producto de su trabajo, motivado por las acertadas decisiones de asignación de recursos y desarrollo de proyectos ambientales por parte de las autoridades.

En el caso de los primíparos electorales, se les presentaron tres artefactos, que fueron evaluados como pertinentes por su sentido lúdico e informativo. Ellos reconocen que la poca formación que reciben en el colegio sobre democracia, es a partir de la memorización de los artículos de la Constitución Nacional, pero como tal no hay una aplicación de estos. Además, afirman que, a pesar de no usar el internet de manera adecuada, quisieran aprender de política si se utilizan medios interactivos donde puedan aplicar lo poco que saben, para así tomar mejores decisiones frente al hecho de votar.

Los Millenials emancipados, fueron muy contundentes al rechazar los artefactos que se les presentaron, alegando que no se trata de mejorar los sistemas de información para conocer las propuestas o planes de gobierno, pues perfectamente pueden encontrarlos en medios virtuales o físicos. El problema, según ellos, está en la actitud de los candidatos, entre su saber ser y saber hacer, lo que no los convence para ejercer la soberanía a partir del voto, o por lo menos tener una afiliación a una ideología con algún partido o movimiento político. El sentir de este grupo, se refleja en la opinión de una mujer de 30 años, profesional en negocios, que expresó: "Sabemos que en Colombia los famosos Millenials pueden decidir el rumbo de un país, porque de acuerdo al DANE la mayor parte de la población colombiana se concentra en edades entre los 20 y 35 años. Pero realmente el problema es la pérdida de la fe que nosotros hemos tenido frente a las últimas formas en las que se ha gobernado este país, en donde prima el interés de unos pocos y el de los demás queda rezagado a un salario mínimo paupérrimo y a unos programas de subsidio mediocres, que generan más pobreza e ignorancia". Lo anterior va de la mano con Martínez-Rosón (2016), quien comprobó que a pesar que los ciudadanos conocen las propuestas de los candidatos, estos pueden llegar a votar por un 
corrupto. El autor asegura que la probabilidad de que se repita este escenario, es menor cuando hay apropiación de más conocimientos políticos, en este caso el ciudadano aprende y perfecciona así su elección.

Las percepciones de los millenials resumen el proceso de aplicación del pensamiento de diseño para reforzar o mejorar las competencias ciudadanas. El individuo no solo requiere estar informado (saber saber), para votar, sino que dentro de su actuar debe integrar la ética a todos los aspectos de su diario vivir (saber ser). Marín-Quintero (2018), lo indica al deducir que algunos profesionales en cargos públicos, se ven involucrados a diario en escenarios de corrupción, donde sus principios cívicos y el comportamiento moral son dejados de lado, dando prioridad a la ambición política o monetaria. Esto para luego entender que comparte un mismo espacio con otros seres humanos, con diferentes ideologías, en donde se autoeduca y a la vez aporta a la formación globalizada de todos ellos (saber hacer), permitiendo así la sinergia del verdadero bien común (Carvajal-Abril, 2016b).

Finalmente, es importante mencionar que esta investigación no se desarrolló de manera tradicional, buscando un antes y un después de la aplicación de una herramienta didáctica. Los modelos de enseñanza y aprendizaje varían con el tiempo y con las circunstancias de las nuevas generaciones. Haber realizado una prueba diagnóstica sobre competencias ciudadanas, para luego medir la incidencia del método utilizado, no era el fin de los investigadores. El mensaje que se promueve con este estudio, es reconocer que hay otras maneras de promover conocimientos en las aulas de clase. Además, que parte fundamental del hacer académico, es la incorporación del uso de la creatividad y el desarrollo de experiencias por parte de los estudiantes, para que se empoderen de lo que el entorno puede ofrecerles.

\section{Conclusiones}

En respuesta a la pregunta problema, efectivamente a través del pensamiento de diseño, se pueden mejorar las competencias ciudadanas de los estudiantes del programa de Administración de Empresas, de la Universidad de la Amazonia. Lo anterior se justifica, porque: i) los estudiantes se apropian de los principios constitucionales para crear un nuevo producto; es decir, generan vivencias con respecto a su realidad como ciudadanos; y ii) con la aplicación de esos principios, se puede fomentar el aprendizaje de la habilidad de pensamiento social en las demás personas, para generar conciencia ciudadana.

La formación en competencias ciudadanas en estudiantes universitarios, debe salirse de los esquemas tradicionales de enseñanza, en donde prima la memoria y la acumulación de datos de orden legal. El objetivo de la formación en ciudadanía, hoy en día, no está en hacer que los estudiantes reciten cada artículo de la constitución o las normas vigentes, se trata más bien de empoderar al sujeto con dicha información para que la pueda usar en su entorno inmediato. En otras palabras, la metodología de clases magistrales para la adquisición de estas competencias, debería quedar rezagada a menos de un diez por ciento del desarrollo de una asignatura en un periodo dado.

Adicionalmente, la construcción de artefactos no solo permitió que los estudiantes, a través de equipos, entendieran la dinámica del ejercicio al voto popular en Colombia. También se pudo constatar que, con ese conocimiento aplicado, los sujetos en formación adquieren habilidades para trasmitirlas a diferentes tipos de poblaciones o públicos. Es decir, se logra el empoderamiento de competencias comunicativas y emocionales, otras de las habilidades que agrupan el desarrollo ciudadano.

El pensamiento de diseño no solo involucra la capacidad para crear e innovar. Esta también se 
puede utilizar para reforzar el aprendizaje de los universitarios, frente a la aplicación de un conocimiento adquirido dentro de la enseñanza dada por los docentes. Con esta técnica, se logra acercar más al futuro profesional al entorno, para que sea capaz de crear su proyecto de vida en donde tenga más opciones de éxito, en cuanto a: lo laboral, lo personal y lo social. La mejor manera de reconocer un nivel de actitud favorable en competencias ciudadanas, es producto del desarrollo de estrategias que van más allá de la enseñanza magistral de los temas del currículo formal, y que incluye otros procesos, como: i) la vida universitaria (actividades extracurriculares, debates, discusiones), y ii) los procesos culturales del contexto y el momento (Álvarez-Rodríguez, Valencia-Serna \& López, 2019).

Finalmente, se da apertura a nuevas sublíneas de investigación dentro de la línea existente en el programa, denominada Educación y Administración: i) técnicas de innovación como apoyo para la formación en ética y responsabilidad social, y ii) mejoramiento de las competencias comunicativas a partir de la elaboración de artefactos, como posibles emprendimientos educativos y/o sociales. Es importante recalcar que los estudiantes participantes en este proceso investigativo, presentarán el examen Saber Pro durante el año 2020. Con estos resultados, se planea una segunda fase de la investigación, que podría articular la actualización curricular que actualmente adelanta el programa en la universidad objeto de estudio, brindando la oportunidad de afianzar todos los componentes de las competencias ciudadanas.

\section{Referencias}

Acosta-Varela, N. (2016). Análisis: competencias de ciudadanía en la Universidad Autónoma de Ciudad Juárez (UACJ). Revista Atenas, 4 (36), 1-10. Recuperado de: http://www.redalyc.org/articulo. oa?id=478055146014

Álvarez-Rodríguez, A. A., Valencia-Serna, A., \& López, H. (2019). Competencias ciudadanas en sedes regionales de la Universidad del Valle: avances y dificultades en el proyecto de formación ciudadana. Revista Boletín Redipe, 8 (4), 44-1. Doi: 10.36260/rbr.v8i4.727

Andrade-Adaime, M. C., Pardo-Rozo, Y. Y., Peña-Torres, P., Marlés-Betancourt, C., Correa-Cruz, L., \& Hermosa-Guzmán, D. (2019). La formación en administración de empresas, Análisis de Contexto. Programa de Administración de Empresas. (Documento en construcción) Universidad de la Amazonia.

Asociación Colombiana de Facultades de Administración, ASCOLFA. (2018). Boletín Estadístico - Investigativo. Observatorio en Ciencias Administrativas. Ascolfa. Recuperado de: http://www. ascolfa.edu.co/observatorio/boletin1.pdf

Benedicto, J., \& Morán, M. L. (2002). La construcción de una ciudadanía activa entre los jóvenes. Instituto de la Juventud.

Calderíus-Fernández, M., \& Martínez-Sánchez, N. (2013). La Formación de competencias ciudadanas en las universidades cubanas. Revista Santiago, 3 (132), 687-713. Recuperado de: https://revistas. uo.edu.cu/index.php/stgo/article/view/45

Carvajal, M. (2016a). La pedagogía praxeológica como componente en el proceso de investigación para la formación ciudadana. Educación y Educadores, 19 (3), 416-436. doi: 10.5294/edu.2016.19.3.6

Carvajal-Abril. H. A. (2016b). Educación en bioética y la formación para la ciudadanía. Praxis \& Saber, 7 (13), 223-242. doi: 10.19053/22160159.4173

Constitución Política de Colombia (1991). Gaceta Constitucional No. 116 de 20 de julio de 1991. Bogotá, Colombia.

Córdoba-Cely, C., Bonilla-Mora, H., \& Arteaga-Romero, J. (2016). Artefactos: Resultado de investigación en diseño. Iconofacto, 11 (17), 30 - 52. doi: http://dx.doi.org/10.18566/iconofac.v11n17.a02 
De Sousa-Santos, B. (2005). La universidad en el siglo XXI. Para una reforma democrática y emancipadora de la sociedad. Editorial Laboratorio de Políticas Públicas.

Dorst, K. (2011). The core of 'design thinking' and its application. Design Studies, 32 (6), 521-532.

Frías, M. (2019). Design thinking: ¿Pueden las empresas aprender del diseño?. Ediciones UADEUniversidad Argentina de la Empresa. Recuperado de: https://repositorio.uade.edu.ar/xmlui/ handle/123456789/8281

Henao-Escovar, J., Ocampo-Talero, A. M., Robledo-Gómez, A. M., \& Lozano-Ardila, M. C. (2007). Los grupos juveniles universitarios y la formación ciudadana. Universitas Psychologica, 7 (3), 853-868. Recuperado de: https://revistas.javeriana.edu.co/ index.php/revPsycho/article/view/396

Instituto Colombiano para la Evaluación de la Educación, ICFES. (2018). Marco de referencia para la evaluación de la educación de la prueba de competencias ciudadanas de los Exámenes de Estado para la Evaluación de la Educación Superior, Saber TyT y Saber Pro, y para la Evaluación de la Educación Básica y Media, Saber $5^{\circ}, 9^{\circ}$ y $11^{\circ}$. ICFES.

Kimbell, L. (2001). Designing for Services as One Way of Designing Services. International Journal of Design. 5 (2). 1-12.

Marín-Quintero, A. E. (2018). El sentido de responsabilidad que subyace al ejercicio profesional de los egresados de las universidades tolimenses. Revista Boletín Redipe, 7 (6), 52-57. Recuperado de: https://revista.redipe.org/index.php/1/article/ view/509

Martínez-Rosón. M. M. (2016). Yo prefiero al corrupto: el perfil de los ciudadanos que eligen políticos deshonestos pero competentes. Reis. Revista Española de Investigaciones Sociológicas, 1 (153), 77-92. Recuperado de: http://www.redalyc. org/articulo.oa?id=99746725005

Ministerio de Educación Nacional - MEN (2014). Documento 24. El sentido de la educación inicial: la exploración. Rey Naranjo Editores.

Ministerio de Educación Nacional - MEN (2012). Propuesta de lineamientos para la formación por competencias en educación superior. Recuperado de: http://www.mineducacion.gov.co/1621/articles261332_archivo_pdf_lineamientos.pdf

Pinilla-Díaz, A. V., \& Torres-Azocar. J. C. (2006). De la educación para la democracia a la formación ciudadana: una década de incertidumbres. Editorial Universidad Pedagógica Nacional / Instituto para el Desarrollo Pedagógico y la Investigación Educativa -IPEP

Redon, S. (2016). Una reflexión sobre la escuela pública y la ciudadanía. Revista Interuniversitaria de Formación del Profesorado, 30 (1), 25-35. Recuperado de: http://www.redalyc.org/articulo. oa? id $=27446519003$

Zambrano, E. L. (2018). Teaching Practices in Developing Citizenship Skills. Revista electrónica de investigación educativa, 20 (1), 69-82. 